\title{
The short form of TSLP is constitutively translated in human keratinocytes and has characteristics of an antimicrobial peptide
}

\author{
L Bjerkan $^{1}$, O Schreurs ${ }^{1}$, SA Engen ${ }^{1}$, FL Jahnsen ${ }^{2}$, ES Baekkevold ${ }^{2}$, IJS Blix ${ }^{1,3}$ and K Schenck ${ }^{1}$
}

Thymic stromal lymphopoietin (TSLP) has multifaceted immunological functions ranging from maintenance of tolerance to induction of disease. Two human transcript variants of TSLP are described: a long form (variant 1 ; IfTSLP) consisting of four exons and an alternative, short form (variant 2; sfTSLP) that lacks two exons compared with variant 1. SfTSLP has not been described at the protein level or functionally studied. Here, we demonstrate that the human sfTSLP is the predominant form of TSLP, constitutively expressed at the mRNA and protein level in keratinocytes of oral mucosa and skin and in salivary glands, is released in saliva, and is not regulated in the same manner as the long form. Compared with IfTSLP, sfTSLP exhibits a markedly stronger antibacterial activity. Synthetic sfTSLP did not activate signal transducer and activator of transcription 5 (STAT5) signaling in CD1c ${ }^{+}$dendritic cells nor interfered with STAT5 activation by IfTSLP. SfTSLP may, therefore, act as an antimicrobial peptide in the oral cavity and on the skin to create a defense barrier that aids in the control of both commensal and pathogenic microbes. The results show that the two translational products of the TSLP gene have a different expression and different biological properties, and emphasize the importance of analyzing the two TSLP isoforms separately.

\section{INTRODUCTION}

Thymic stromal lymphopoietin (TSLP) is a cytokine first identified in a mouse model as a B-cell growth factor produced by a thymic stromal cell line. ${ }^{1}$ TSLP is produced by multiple cell types including epithelial and dendritic cells (DCs). ${ }^{2,3}$ Diverse immune cells can express the TSLP receptor and respond to TSLP, including mast cells, DCs, and T cells. ${ }^{2,4,5}$ TSLP may have dual immunoregulatory roles. On the one hand, it has been implicated in the pathogenesis of several disorders, including allergic and asthmatic diseases, autoimmunity, and cancer. ${ }^{6}$ On the other hand, TSLP has been associated with protective and tolerogenic functions. For example, human DCs conditioned with TSLP have been shown to augment intestinal epithelial cell-mediated IgA2 class switching through the induction of a proliferation-induced ligand, indicating a protective role of TSLP in the intestine. ${ }^{7}$ Further, in vitro studies have suggested a role for TSLP in the generation of tolerogenic DCs that induce the differentiation of regulatory $\mathrm{T}$ cells both in the intestine and in the thymus. ${ }^{8-10}$ Finally, TSLP has been shown to exert antimicrobial activity, primarily mediated by the C-terminal region of the protein. ${ }^{11}$

Two transcript variants of human TSLP are described: a long form of TSLP (lfTSLP, variant 1 ) and an alternative, short form (sfTSLP, variant 2) consisting of four and two exons, respectively. LfTSLP has a sequence of 159 amino acids (aa) corresponding to a calculated molecular weight (MW) of $18.1 \mathrm{kDa}$, but the N-terminal amino acids $1-28$ form a signal peptide, leaving a chain with a calculated MW of $15 \mathrm{kDa}$, which corresponds to the MW of commercially available recombinant lfTSLP produced in prokaryotic cells. SfTSLP uses a downstream in-frame start codon compared with variant 1 . The encoded variant 2 is shorter at the $\mathrm{N}$ terminus and fully overlaps the amino-acid sequence of variant 1 in the $\mathrm{C}$-terminal region (National Center for Biotechnology Information, National Library of Medicine, Bethesda, MD). The sequence of sfTSLP shows two potential starting methionines that can give rise to either a 63 or a 60 aa peptide (G3XAM8 and Q96AU7, respectively; http://www.uniprot.org), with calculated MW of

${ }^{1}$ Department of Oral Biology, Dental Faculty, University of Oslo, Oslo, Norway. ${ }^{2}$ Centre for Immune Regulation and Department of Pathology, Medical Faculty, University of Oslo, Oslo, Norway and ${ }^{3}$ Department of Periodontology, Dental Faculty, University of Oslo, Oslo, Norway. Correspondence: K Schenck (karl.schenck@odont.uio.no) 
7.4 and $7.1 \mathrm{kDa}$, respectively. The $\mathrm{N}$-terminal sequence of sfTSLP contains a potential N-terminal 20 aa signal peptide (PSORT; http://psort.hgc.jp), and if this peptide is left out, the calculated size of the remaining sfTSLP peptide lies at $5.2 \mathrm{kDa}$. With a few exceptions, ${ }^{12}$ most studies do not distinguish between these TSLP variants, neither at the mRNA nor at the protein level, and most authors collectively designate these variants as "TSLP." Only the IfTSLP variant has previously been described at the protein level, and thus functional studies of TSLP have been restricted to the investigation of the effects of the full-length TSLP protein.

In this study, we wanted to assess whether sfTSLP is translated, map the expression at the mRNA and protein level in situ, examine the regulation, and test the potential for antimicrobial activity. Focusing on barrier surfaces including the oral mucosa and the skin, we found that sfTSLP is the predominant form of TSLP expressed in keratinocytes at steady state, both at the mRNA and protein levels. SfTSLP was constitutively expressed and only slightly upregulated in response to inflammatory factors, in marked contrast to IfTSLP. SfTSLP showed antimicrobial activity and the effect was markedly stronger than that of lfTSLP. SfTSLP was expressed in salivary glands and detected in saliva, whereas lfTSLP was not. Therefore, a function of sfTSLP may be to act as an antimicrobial peptide (AMP) at barrier surfaces.

\section{RESULTS}

\section{Transcription and translation of sfTSLP}

To investigate the mRNA expression of the two variants of human TSLP separately, we used variant-specific primers recognizing sfTSLP and lfTSLP, respectively. Lysates were made from whole oral mucosal biopsies and cultures of primary oral keratinocytes, and mRNA expression was determined by real-time polymerase chain reaction. We found that sfTSLP
mRNA was the predominant form of TSLP expressed in oral mucosa and primary oral keratinocytes (Figure 1a). The same pattern was seen in the skin (D Khnykin et al., unpublished data).

The finding of constitutive sfTSLP mRNA expression prompted us to investigate whether sfTSLP was also expressed at the protein level because tissue expression of sfTSLP protein has not previously been reported. Antibodies prepared with recombinant full-length TSLP as an immunogen recognize both isoforms (Supplementary Figure S1 online, left) because the sfTSLP protein sequence completely overlaps the IfTSLP sequence in the C-terminal region. Hence, production of sfTSLP-specific antibodies is not possible and we therefore chose an indirect approach: sfTSLP reactivity was removed from polyclonal anti-full-length TSLP antibody, by incubating the antibody with sfTSLP coupled to CNBr-activated Sepharose $4 \mathrm{~B}$ beads. Thus, an sfTSLP-absorbed product that only reacted with IfTSLP was obtained, which allowed us to compare the total content of TSLP protein (long and short combined) with lfTSLP-specific activity (Supplementary Figure S1, right). After sodium dodecyl sulfate-polyacrylamide gel electrophoresis and western blotting of lysates of oral mucosal epithelial sheets and primary oral epithelial cells, immunoreactivity was detected at $9 \mathrm{kDa}$ with the unabsorbed antibody preparation (Figure 1b, left), which were not visible after incubation with the sfTSLP-absorbed antibody preparation (Figure $\mathbf{1 b}$, middle). This indicated the presence of sfTSLP in the samples. In accordance with the mRNA results, no lfTSLP protein was detected in either primary oral keratinocytes or oral mucosal epithelial sheets because no band was seen at the expected position of about $23 \mathrm{kDa}^{13}$ (compare with Figure 1f, right). For additional validation of the specificity of the $9 \mathrm{kDa}$ band, cultured oral keratinocytes were transfected with endoribonuclease-prepared siRNA (esiRNA) targeting

Figure 1 Transcription and translation of short form of thymic stromal lymphopoietin (sfTSLP). (a) Relative mRNA expression of long form of TSLP (IfTSLP) and sfTSLP in oral mucosal biopsies (left, $n=4$ ) and cultured oral keratinocytes (KCs) (right; $n=4$ ). Bars indicate median values. *Statistically significant difference, paired $t$-test. (b) Western blots showing TSLP protein expression in lysates of oral mucosal epithelial sheets (ES; $n=4)$ and cultured oral KCs $(n=4)$ by use of either polyclonal anti-TSLP antibody (TSLP pAb) (ab47943) (left), with the same antibody absorbed with sfTSLP peptide (sf abs TSLP pAb; middle) or with rabbit immunoglobulin G (IgG) (isotype control, right; $n=3$ ). Numbers indicate the positions of the molecular weight markers. Black arrowhead indicates position of sfTSLP. (c) Expression of sfTSLP mRNA (left) and protein (right, $n=5$ ) in cultured oral keratinocytes transfected with scramble endoribonuclease-prepared siRNA (esiRNA) or with TSLP-specific esiRNA. Band density of detected bands in the scrambled control and TSLP esiRNA-treated samples (right, mean \pm s.d.; normalized to scrambled control). *Statistically significant difference, paired $t$-test. (d) Immunohistochemical staining (brown color) of sections of oral mucosa and skin by use of either TSLP pAb (ab47943) (far left) or the same antibody absorbed with sfTSLP peptide (middle left; representative stainings, $n=10$ for oral mucosa, $n=5$ for skin). Counterstaining with hematoxylin. In situ hybridization by use of sfTSLP-specific probe (middle right; representative stainings, $n=5$ for oral mucosa, $n=3$ for skin) or scramble mRNA probe (far right) on sections of oral mucosa and skin. Counterstaining with nuclear fast red. Pictures were taken at an original magnification of $\times 20$. Bar $=100 \mu \mathrm{m}$. (e) Relative mRNA expression of sfTSLP ( $n=8$ for polyinosinic-polycytidylic acid (poly(I:C)), $n=5$ for interferon- $\gamma$ (IFN- $\gamma)$ and tumor necrosis factor- $\alpha$ (TNF- $\alpha$ )/interleukin-1 $\beta$ (TNF $\alpha / \mathrm{IL}-1 \beta)$ ) and IfTSLP $(n=9$ for poly $(\mathrm{I}: \mathrm{C}), n=6$ for IFN- $\gamma$ and TNF $\alpha / \mathrm{IL}-1 \beta)$ in cultured oral KCs in response to the indicated stimuli. Cells were stimulated for $4 \mathrm{~h}$ and overnight $(\mathrm{ON})$. Bars indicate median values. * Statistically significant difference, repeatedmeasures analysis of variance on ranks with pairwise multiple comparison. (f) Expression of sfTSLP at $9 \mathrm{kDa}(\mathrm{ab} 47943)(\mathrm{left}, n=3)$ and lfTSLP at $23 \mathrm{kDa}$ (no. 4021) (right, $n=4$ ) protein in response to poly $(\mathrm{I}: \mathrm{C})$ for $4 \mathrm{~h}$ and $\mathrm{ON}$ in cultured oral keratinocytes. Colored numbers and colored arrows indicate the positions of the molecular weight standards. Black numbers and black arrows indicate the positions of sfTSLP and IfTSLP. (g) Immunohistochemical staining (brown color) of sections of oral mucosa exposed to smokeless tobacco "snus," by use of either TSLP pAb (ab47943) or the same antibody absorbed with sfTSLP peptide (representative stainings, $n=5$ ). Counterstaining with hematoxylin. Pictures were taken at an original magnification of $\times 10$. Bar $=100 \mu \mathrm{m}$. sf abs TSLP pAb, sfTSLP absorbed anti-TSLP pAb. (h) Immunohistochemical staining (brown color) of sections of submandibular gland made by use of either TSLP pAb (ab47943) (left) or the same antibody absorbed with sfTSLP peptide (middle) ( $n=3)$. Counterstaining with hematoxylin. In situ hybridization sfTSLP-specific probe (right; blue color; $n=3$ ). Counterstaining with nuclear fast red. Pictures were taken at an original magnification of $\times 20$. Bar $=100 \mu \mathrm{m}$. (i) Detection of sfTSLP in saliva by western blotting. A specific band at $9 \mathrm{kDa}$ (black arrow) was detected by use of TSLP pAb (no. 4021) (left), which was not visible after use of the same antibody absorbed with sfTSLP peptide (right) ( $n=8)$. Colored numbers and colored arrows indicate the positions of the molecular weight standards. 
full-length TSLP mRNA, to reduce the level of sfTSLP mRNA (Figure 1c, left). Lysates from the transfected cells showed a significantly weakened $9 \mathrm{kDa}$ peptide band as compared with that obtained with scrambled controls (Figure 1c, right), confirming that this band corresponds to
sfTSLP. The band detected at $9 \mathrm{kDa}$ diverges from the possible calculated sizes of 7.4 and $7.1 \mathrm{kDa}$, or $5.2 \mathrm{kDa}$ without potential leader sequence, of the amino-acid sequence of sfTSLP. This shift is probably due to posttranslational modifications (PTMs) of sfTSLP.
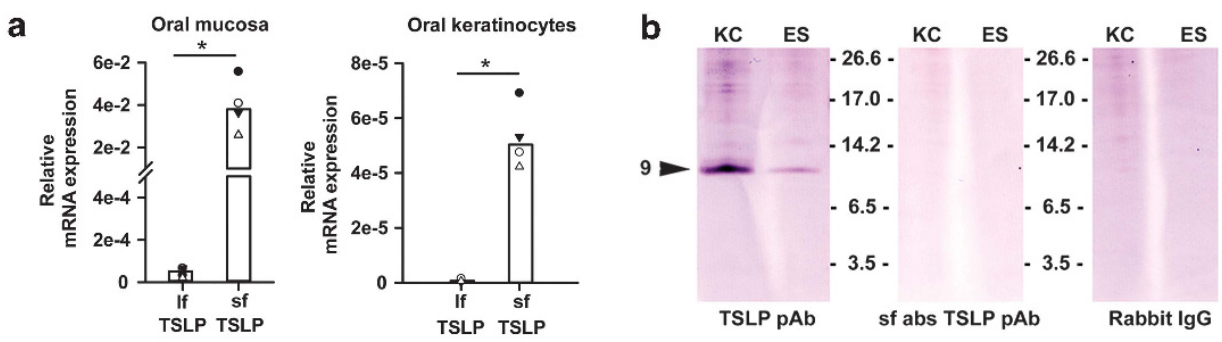

d

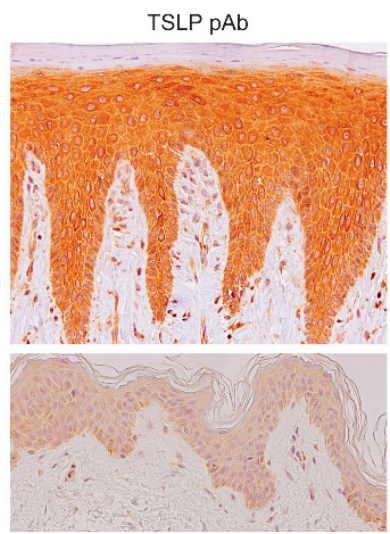

e

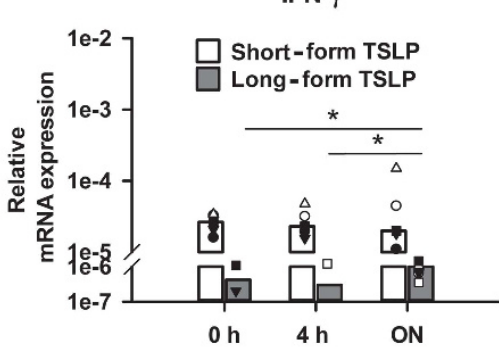

f
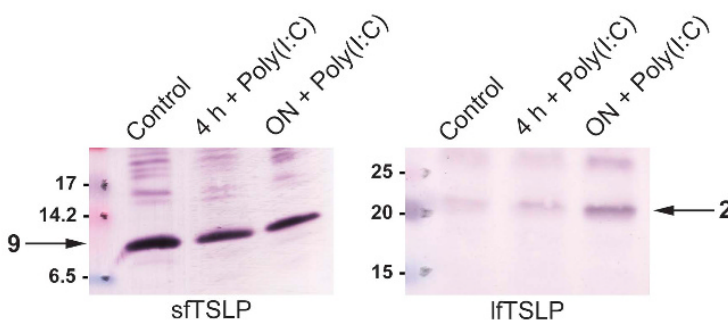

h

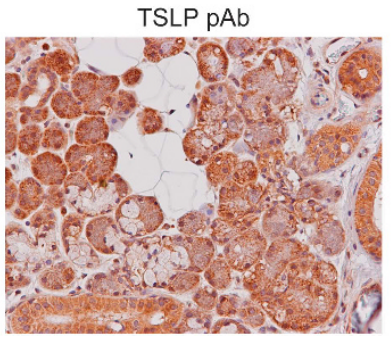

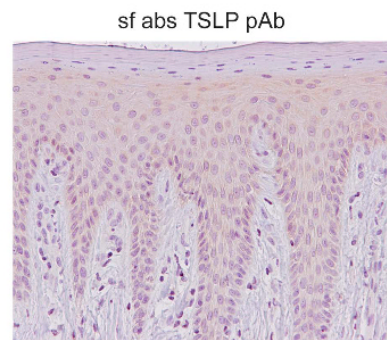
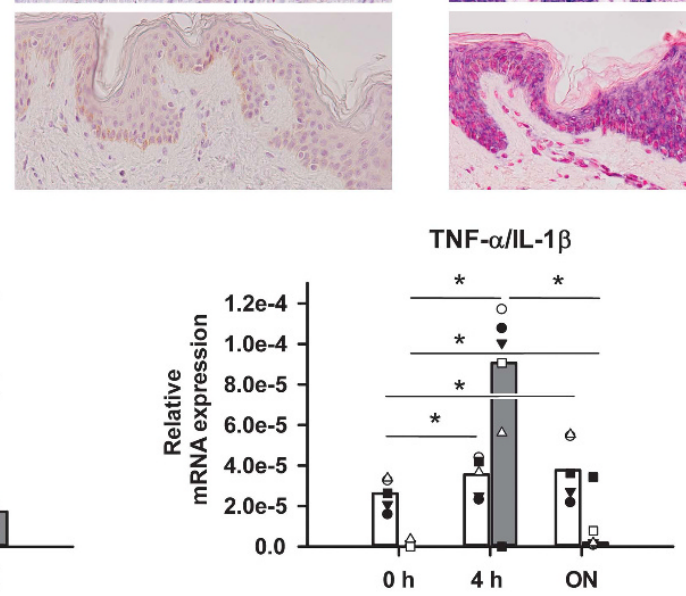

g

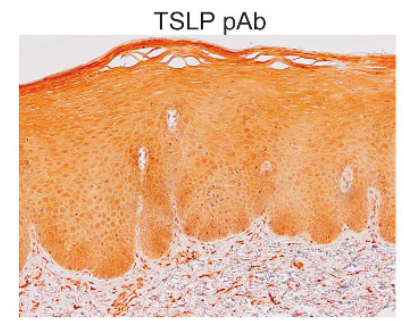

sfTSLP mRNA-specific probe
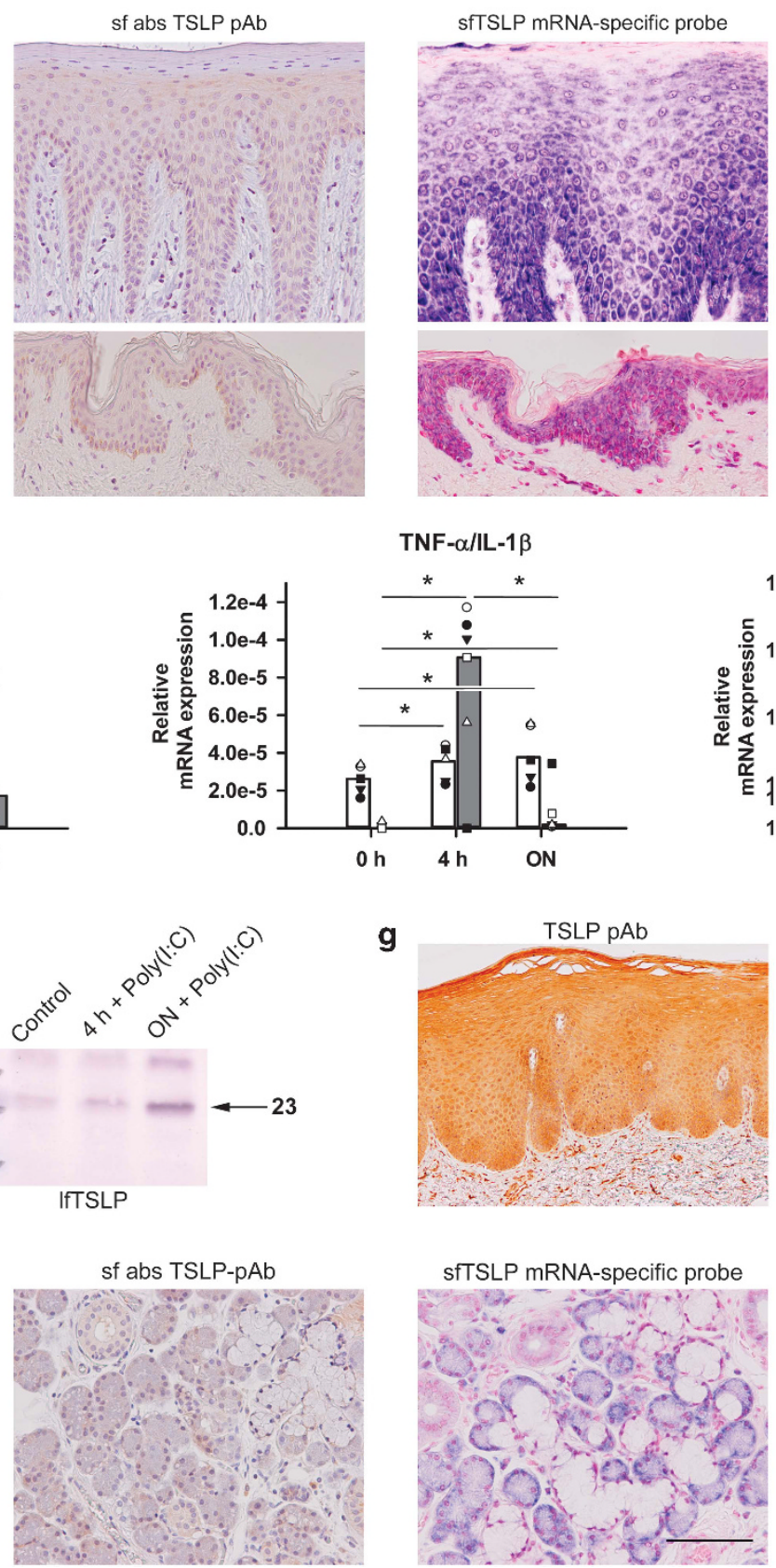
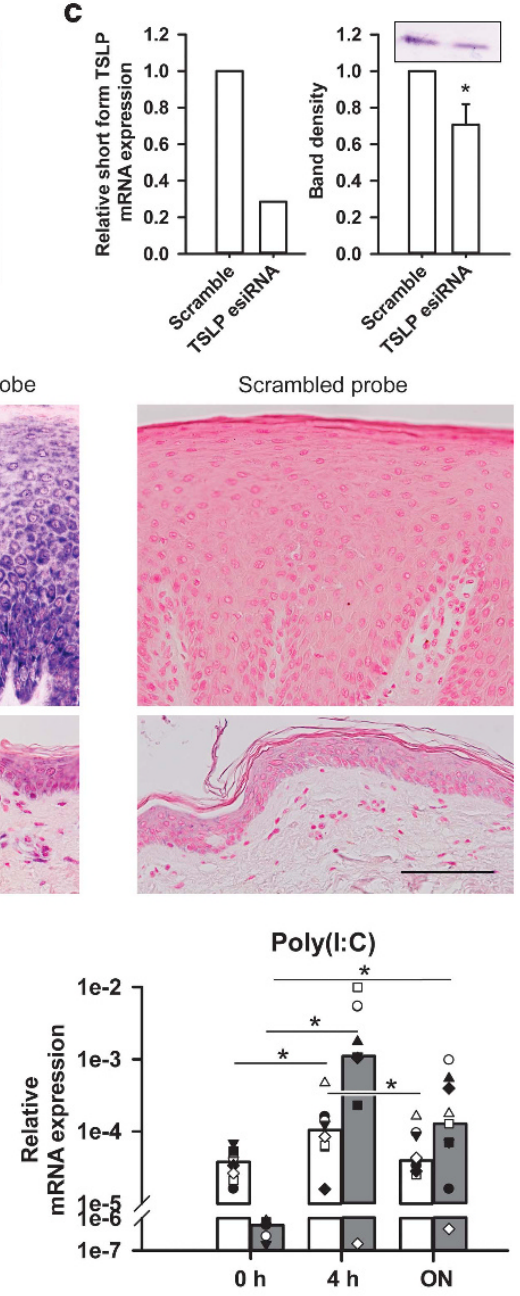

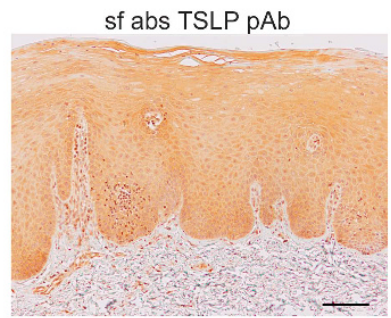

i

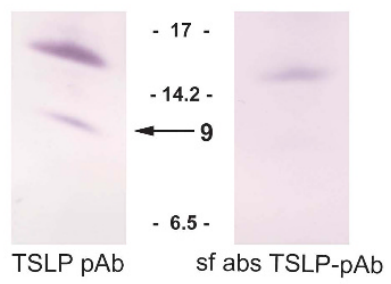



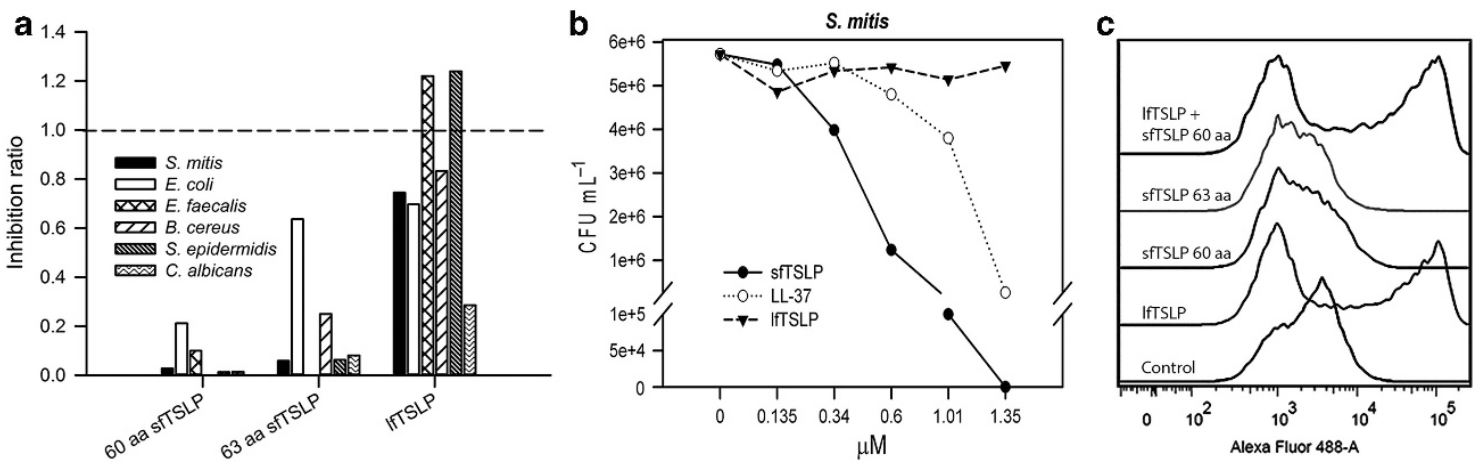

Figure 2 Antimicrobial activity of short and long forms of thymic stromal lymphopoietin (sf and IfTSLP) and the effect of sfTSLP on signal transducer and activator of transcription 5 (STAT5) phosphorylation of CD1c + dendritic cells. (a) Suspensions of the indicated bacterial and fungal species were treated for $2 \mathrm{~h}$ with 60 amino acid (aa) sfTSLP, and 63 aa sfTSLP or IfTSLP peptide at a concentration of $1.35 \mu \mathrm{m}$ before being plated on agar. Colony-forming units per $\mathrm{ml}$ was determined after incubation overnight. The values were normalized to the levels obtained without the addition of test peptides (broken line). Shown is a representative experiment (sfTSLP: $n=3$ for all microbes except $S$. mitis and $C$. albicans, where $n=5$; IfTSLP: $n=1$ for $B$. cereus; $E$. coli and $E$. faecalis, $n=2$ for $S$. mitis, S. epidermidis, and $C$. albicans). (b) Suspensions of $S$. mitis were treated with equimolar concentrations of 60 aa sfTSLP, LL-37, or IfTSLP and analyzed as in a. (c) STAT5 phosphorylation in response to IfTSLP, 60 aa sfTSLP, 63 aa sfTSLP or IfTSLP combined with sfTSLP in blood-derived $\mathrm{CD}_{1 \mathrm{c}}{ }^{+}$myeloid DCs incubated with polyinosinic-polycytidylic acid (poly(l:C)) for $24 \mathrm{~h}$, and then treated with sfTSLP or/and IfTSLP for $15 \mathrm{~min}$. Phosphorylation of STAT5 was assessed by flow cytometry. Shown is a representative of three individual experiments.

Having demonstrated the protein expression of sfTSLP in western blots, we next carried out TSLP isoform-specific staining by immunohistochemistry. By comparing stainings of sections of oral mucosa and skin with the sfTSLP-absorbed antibody preparation and the unabsorbed anti-TSLP antibody, we found that sfTSLP protein was expressed in all epithelial cell layers of clinically healthy oral epithelium and skin epidermis, while no lfTSLP was seen (Figure 1d). SfTSLP mRNA expression was detected at the same sites with in situ hybridization (Figure 1d). We also investigated isoform-specific expression of TSLP in oral mucosal lesions induced by the use of the smokeless tobacco "snus." In these lesions, IfTSLP peptide was found to be upregulated in the epithelial cells (Figure 1g). This constituted a positive staining control for the present immunochemical staining method for lfTSLP protein.

To investigate how the two TSLP variants are regulated under inflammatory conditions, cultured oral keratinocytes were stimulated with proinflammatory factors, including interferon- $\gamma$ (IFN- $\gamma$ ), tumor necrosis factor- $\alpha$ (TNF- $\alpha$ )/ interleukin-1 $\beta$ (IL-1 $\beta$ ) and polyinosinic-polycytidylic acid (poly(I:C)). In such cells, IfTSLP mRNA was upregulated after $4 \mathrm{~h}$ exposure to TNF- $\alpha / \mathrm{IL}-1 \beta$ and poly(I:C), and after $24 \mathrm{~h}$ exposure to all stimuli (Figure 1e). Poly(I:C) and TNF- $\alpha / \mathrm{IL}-1 \beta$ stimulation also upregulated sfTSLP mRNA after $4 \mathrm{~h}(P<0.05)$, but when compared with lfTSLP, the changes were modest. At the protein level, lfTSLP was upregulated after $24 \mathrm{~h}$ in response to poly(I:C), whereas upregulation of sfTSLP protein was not detected (Figure 1f).

\section{SfTSLP exerts potent antimicrobial activity and does not induce STAT5 signaling}

Full-length recombinant TSLP displays antimicrobial activity against bacteria and yeasts. ${ }^{11}$ We therefore examined whether the antimicrobial activity was retained in the sfTSLP peptide and compared it with the activity of lfTSLP, using a panel consisting of diverse bacterial and fungal species. SfTSLP (60 or $63 \mathrm{aa}$ ) or IfTSLP were added to microbial suspensions for $2 \mathrm{~h}$ and then plated and incubated overnight. Colonies were counted the next day and colony-forming units per $\mathrm{ml}$ were determined. The results showed that sfTSLP exerted potent antimicrobial activity against all the tested species (Figure 2a). Addition of polyclonal anti-TSLP antibody to sfTSLP (ab47943; Abcam), before it was incubated with Streptococcus mitis, reduced the antimicrobial activity by about half, showing that the reduction in colony-forming units per ml was specifically due to the action of sfTSLP (data not shown). Dose-response curves using S. mitis showed that the effect of sfTSLP was stronger than that obtained by the well-characterized AMP LL-37 (Figure 2b).

LfTSLP induces STAT5 signaling in DCs. ${ }^{6}$ Neither 60 - nor 63 aa sfTSLP induced this activation in poly(I:C)-stimulated CD1 $\mathrm{c}^{+}$DCs, nor interfered with STAT5 activation by lfTSLP (Figure 2c).

\section{SfTSLP is produced in salivary glands and is released in saliva}

Having demonstrated an antimicrobial role of sfTSLP, we investigated the expression of the peptide in salivary glands and whether it can be detected in saliva. There are three major pairs of salivary glands (the parotid, submandibular, and sublingual glands), in addition to the minor salivary glands scattered in the submucosa throughout the oral and oropharyngeal cavities. By comparing immunochemical stainings carried out with the unabsorbed anti-TSLP antibody with the sfTSLP-absorbed antibody preparations, we found that SfTSLP was expressed in all four gland types, whereas lfTSLP was not detected (Figure $1 \mathrm{~h}$ and Supplementary Figure S2). In the submandibular gland, in which the acini are both mucous and serous, sfTSLP was expressed in both acini and ducts (including intercalated, striated, and collecting ducts) (Figure 1h). 
Production of sfTSLP mRNA was detected by in situ hybridization with an sfTSLP-specific probe (Figure $\mathbf{1 h}$ and Supplementary Figure S2) and supported the observation of sfTSLP protein expression. In the ducts and serous and mucous acini of all glands, both sfTSLP protein and mRNA were detected in varying degrees (Supplementary Figure S2).

We finally investigated the presence of sfTSLP in salivary secretions. Whole, unstimulated saliva was taken from healthy volunteers and subjected to sodium dodecyl sulfate-polyacrylamide gel electrophoresis and western blotting, and a band was detected at $9 \mathrm{kDa}$ (Figure 1i) with the unabsorbed anti-TSLP antibody, which was not visible with the sfTSLP-absorbed antibody, indicating the presence of sfTSLP in saliva.

\section{DISCUSSION}

The epithelial barrier is the first line of defense against microbial invaders and foreign antigens. TSLP has been shown to be produced by epithelial cells and to promote immune responses that can be protective or detrimental to the host. ${ }^{2,14}$ Most studies have, however, not taken into account that there are two variants of TSLP, but one investigation of a panel of human tissues showed that sfTSLP mRNA was constitutively found at many body sites, whereas lfTSLP mRNA was not seen. ${ }^{12}$ The present study shows that sfTSLP mRNA is the predominant form of TSLP expressed in oral mucosa and primary oral keratinocytes, which is in parallel with previous observations at other body sites. ${ }^{12}$ Expression of sfTSLP peptide was detected by western blotting of lysates from oral mucosal epithelial sheets and primary oral epithelial cells as a band at $9 \mathrm{kDa}$, of which the identity was confirmed by esiRNA knockdown in oral keratinocyte cultures. When the entire gene sequence coding for sfTSLP is translated into amino-acid sequence, the possible calculated sizes of sfTSLP are 7.4 or $7.1 \mathrm{kDa}$, or $5.2 \mathrm{kDa}$ when a potential signal peptide is left out of the equation. No specific bands were detected at any of those positions. The shift to $9 \mathrm{kDa}$ is likely to be due to PTMs of sfTSLP, possibly glycosylation, because the sfTSLP amino-acid sequence contains a potential glycosylation site ${ }^{15}$ and PTM, probably glycosylation, is seen for IfTSLP, which has a calculated molecular size of $15 \mathrm{kDa}$ but appears at $23 \mathrm{kDa}$ in western blotting. ${ }^{13}$ In situ examination by immunohistochemistry and in situ hybridization concurred with the quantitative real-time PCR and western blotting results: constitutive expression of sfTSLP was seen, while no lfTSLP was detected. As TSLP expression has been shown to be increased in airway smooth muscle cells following exposure to cigarette smoke extract, ${ }^{16}$ we investigated the isoform-specific expression of TSLP in oral mucosal lesions induced by the use of another toxic tobacco derivative that is applied to the oral mucosa by users, the smokeless tobacco "snus." LfTSLP peptide was found to be upregulated in the epithelial cells of these biopsies. This thus accorded with the observations in cigarette smoke extract-exposed airway tissue ${ }^{16}$ and in addition constituted a positive staining control for our immunochemical staining method for lfTSLP protein. Our findings, showing that the sfTSLP transcript is expressed at the protein level and is constitutive in certain types of cells, may necessitate revisiting of parts of the literature on TSLP. Most of the available antibody preparations against TSLP have unspecified variant specificity and may recognize both isoforms, and as long as it is unknown which isoform is detected, the interpretation of results remains highly imprecise.

Stimulation of cultured oral keratinocytes with proinflammatory factors, including IFN- $\gamma$, TNF- $\alpha / \mathrm{IL}-1 \beta$, and poly(I:C), showed significant upregulation of IfTSLP mRNA, while only slight changes were seen for sfTSLP. This is in accordance with earlier studies that showed constitutive sfTSLP but no lfTSLP mRNA expression in human bronchial epithelial cells, and upregulation of lfTSLP but not sfTSLP mRNA after exposure to poly(I:C). ${ }^{12}$ The observation that sfTSLP is not regulated to the same degree as IfTSLP in oral keratinocytes in response to inflammatory stimuli indicates that sfTSLP serves purposes other than lfTSLP.

The body surfaces are in permanent contact with an array of diverse microorganisms and are colonized by a complex microbiota. Despite these permanent threats, the epithelium is mostly able to shield the body from microbial invasion. One of the defense strategies used by the epithelia at mucosal and skin surfaces is the production of AMPs. ${ }^{17}$ Common features for many AMPs are cationicity and $\alpha$-helical confirmation, ${ }^{18}$ characteristics that also apply to TSLP. ${ }^{11}$ Peptides derived from full-length TSLP display antimicrobial activity against bacteria and yeasts, with the antimicrobial effect located in a 34 aa peptide spanning the C-terminal region of full-length TSLP. ${ }^{11}$ We therefore examined whether the antimicrobial activity was retained in the sfTSLP peptide and compared it with the activity of lfTSLP, using a panel consisting of diverse bacterial species: $S$. mitis and Staphylococcus epidermidis are components of the commensal flora of the oral cavity ${ }^{19}$ and the skin ${ }^{20}$ respectively, Enterococcus faecalis ${ }^{21}$ and Escherichia coli $^{22}$ are found within the commensal flora of the gastrointestinal tract, and Bacillus cereus is a Gram-positive spore-forming bacterial species able to cause food poisoning and opportunistic infections. ${ }^{23} \mathrm{We}$ found that synthetic sfTSLP exerted potent antimicrobial activity against all the tested bacterial species. The effect was markedly stronger than that obtained with lfTSLP, which in our test system showed lower antimicrobial activity against S. mitis, E. coli, and B. cereus as compared with sfTSLP, and no detectable activity against $E$. faecalis and S. epidermidis. This means that the antimicrobial activity was retained within the sfTSLP peptide and was not dependent on the presence of complete full-length peptide. LfTSLP can be cleaved by Staphylococcus aureus protease, among others in a C-terminal 34 aa fragment, displaying a strong antimicrobial activity. ${ }^{11}$ It is conceivable that bacterial proteases also can degrade sfTSLP to this shorter fragment, or that, in fact, the naturally occurring form of this fragment corresponds to sfTSLP.

The surfaces of the oral cavity are covered by secretions produced by the salivary glands. Saliva represents an important ingredient in oral homeostasis through its participation in functions such as lubrication, digestion, wound healing, and antimicrobial defense. Several AMPs are expressed and secreted 
by salivary glands, including LL-37, and $\alpha$ - and $\beta$-defensins, and contribute to the protection of oral mucosal surfaces. ${ }^{24}$ SfTSLP peptide and mRNA were detected by immunohistochemistry and in situ hybridization, respectively, in parotid, submandibular, sublingual, and labial salivary glands. By western blotting, sfTSLP peptide was detected in saliva at $9 \mathrm{kDa}$, a size similar to that obtained after processing lysates from cultured oral keratinocytes. This is different from the 7.1 and $7.4 \mathrm{kDa}$ sizes of the peptides that were used to examine the antimicrobial function of sfTSLP, and therefore salivary sfTSLP also may have undergone PTM. PTM can affect functionality of proteins and the sfTSLP in saliva may, therefore, not necessarily have the same effect as the synthetic peptide used in the antimicrobial assays. This hampers the interpretation of the function of-in this case-sfTSLP, as it does for lfTSLP, where this issue also is unexplored: most in vitro research on IfTSLP has used $15 \mathrm{kDa}$ recombinant lfTSLP produced in prokaryotes, while the naturally occurring TSLP in human cells or tissues appears to have an estimated MW of $22-24 \mathrm{kDa}$, as detected in the present and other studies. ${ }^{13,25,26}$ The effect of PTM on the function of both sfTSLP and lfTSLP, therefore, deserves further investigation, but the present observations still indicate that sfTSLP may act as an AMP in the oral cavity and skin to create a defense barrier that aids in the control of both commensal and pathogenic microbes. Whether levels of sfTSLP can reach active antimicrobial concentrations is, similar to other AMPs detected in saliva, difficult to determine because saliva is a highly complex secretion that contains an array of other AMPs that interfere with specific antimicrobial assays. ${ }^{24}$

In summary, the current study shows that sfTSLP is the predominant form of TSLP, widely and constitutively expressed at the protein level in oral and skin keratinocytes and in salivary glands, is present in saliva, and has antimicrobial activity. The function of sfTSLP in the oral cavity and in the skin may be to control microbial invaders. Of note, the fact that sfTSLP is transcribed and translated makes it mandatory to analyze the two TSLP variants separately.

\section{METHODS}

TSLP peptides and anti-TSLP antibodies. Full-length (132 aa; sequence: MYDFTNCDFEKIKAAYLSTISKDLITYMSG-TKSTEFNN TVSCSNRPHCLTEIQSLTFNPTAGCASLAKEMFAMKTKAALAIW CPGYSETQINATQAMKKRRKRKVTTNKCLEQVSQLQGLWRRFN RPLLKQQ) recombinant TSLP was obtained from Peprotech (Rocky Hill, NJ). Synthetic sfTSLP peptides (63 aa: MFAMKTKAALAIWC PGYSETQINATQAMKKRRKRKVTTNKCLEQVSQLQGLWRRFNR PLLKQQ and 60 aa: MKTKAALAIWCPGYSETQINATQAMKK RRKRKVTT-NKCLEQVSQLQGLWRRFNRPLLKQQ) were customprepared by ProteoGenix SAS (Schiltigheim, France). Antibodies reactive with TSLP were ab47943 (Abcam, Cambridge, MA; $0.5 \mu \mathrm{g} \mathrm{ml}^{-1}$ ), nos. 4021 and 4023 (both from Pro-Sci, Poway, CA). LfTSLP-specific antibody preparations were made by absorbing ab47943 or no. 4021 with 60 aa sfTSLP peptide as follows. CNBr-activated Sepharose 4B beads (Sigma-Aldrich, St Louis, MO) were prepared and coupled to 60 aa sfTSLP protein (ProteoGenix SAS) following the manufacturer's protocol. Twenty micrograms of sfTSLP protein was used per $250 \mathrm{mg}$ beads. SfTSLP-coupled beads were suspended in $1 \%$ bovine serum albumin in phosphate-buffered saline (PBS) with $\mathrm{NaN}_{3}$ and incubated with rabbit polyclonal anti-TSLP antibody (ab47943 or no. 4021) at
$4{ }^{\circ} \mathrm{C}$ overnight. Supernatant, consisting of lfTSLP-specific antibodies, was collected. The preparation recognized lfTSLP but not sfTSLP (Supplementary Figure S1).

Biopsy material and collection of saliva. Oral mucosal biopsies were obtained from healthy volunteers undergoing third molar tooth extraction in private oral surgery clinics (see Acknowledgments). Biopsies from leukoplakia at oral sites with that were chronically exposed to the smokeless tobacco "snus" were obtained as part of histological diagnosis. Biopsies of parotid salivary glands were collected from healthy volunteers undergoing orthognatic surgery by retromandibular approach, and biopsies of submandibular glands and sublingual salivary glands were collected from patients undergoing surgery, including removal of the entire or parts of the glands, at the Oslo University Hospital, Oslo, Norway. Labial glands were collected from healthy volunteers by excision at the Department of Oral Surgery and Oral Medicine, Faculty of Dentistry, University of Oslo, Norway or at the Oslo University Hospital, Oslo, Norway. Whole resting saliva was collected in sterile plastic tubes during a $5 \mathrm{~min}$ period from healthy volunteers recruited at the Department of Oral Biology, Faculty of Dentistry, University of Oslo, Norway. The study was carried out according to the Helsinki Declaration's principles for biomedical research and was approved by the Ethical Committee of Health (REK $S \varnothing R$, Norway). Written informed consent was obtained from all donors.

Cell culture. Primary oral keratinocytes were isolated from oral mucosal biopsies according to an established protocol. ${ }^{27}$ The epithelial sheet was removed from the connective tissue of the biopsy after dispase treatment $\left(4^{\circ} \mathrm{C}\right.$, overnight $)$ and cut into small pieces, which were then treated with trypsin-EDTA (Invitrogen, Paisley, UK) for $7 \mathrm{~min}$ at $37^{\circ} \mathrm{C}$. The treatment was stopped by the addition of fetal bovine serum (Cambrex, Verviers, Belgium). Cells were cultured in keratinocyte serum-free medium (17005; Gibco-BRL, Gaithersburg, MD) supplemented with $1 \mathrm{ng} \mathrm{ml}^{-1}$ human recombinant epidermal growth factor, $25 \mu \mathrm{g} \mathrm{ml}^{-1}$ bovine pituitary extract (both from Gibco-BRL) and $1 \%$ penicillin, $1 \%$ streptomycin, and $0.25 \mathrm{mg} \mathrm{ml}^{-1}$ amphotericin B (Lonza, Basel, Switzerland) in $25-\mathrm{cm}^{2}$ cell-culture flasks (Nalge Nunc, Rochester, NY). Keratinocytes were passaged 1:4 by trypsinization. Cells were maintained in a humidified atmosphere of $5 \% \mathrm{CO}_{2}$ in air at $37^{\circ} \mathrm{C}$. Cell counting was carried out with a Coulter Counter Z2 (Beckman Coulter, Fullerton, CA). Cells were used between second and fifth passages. Cells $\left(4 \times 10^{5}\right.$ for stimulation experiments or $3 \times 10^{5}$ for esiRNA experiment per well) were plated in $3 \mathrm{ml}$ of keratinocyte serum-free medium, in 6-well plates. Stimulation experiments were performed at approximately $80 \%$ confluency and esiRNA experiments were performed at approximately $50 \%$ confluency. Cells were stimulated with either $50 \mathrm{ng} \mathrm{ml}^{-1} \mathrm{TNF}-\alpha$, $10 \mathrm{ng} \mathrm{ml}^{-1}$ IL-1 $\beta, 10 \mathrm{ng} \mathrm{ml}^{-1}$ IFN- $\gamma$ (all from Peprotech), or $10 \mu \mathrm{g} \mathrm{ml}^{-1}$ poly(I:C) (Sigma-Aldrich). The wells were washed with ice-cold PBS before harvesting. For western blotting, cells were harvested by scraping in Iscove's modified Dulbecco's medium (Sigma-Aldrich) containing 10\% FBS, centrifuged, and washed once in ice-cold PBS before lysis with $0.5 \%$ NP-40 (Millipore, Merck Chemicals, Nottingham, UK) in PBS supplemented with protease inhibitor cocktail and EDTA (Pierce Biotechnology, Rockford, IL) for $30 \mathrm{~min}$ on ice. Lysates were centrifuged at $16,000 \mathrm{~g}$ and the supernatants were used for further analyses. For RNA extraction, cells were lysed directly in $350 \mu \mathrm{l}$ RLT buffer containing $\beta$-mercaptoethanol (Qiagen, Valencia, CA) in the well. RNA was extracted from oral mucosal biopsies by cutting biopsies into small pieces, adding $350 \mu \mathrm{l}$ of RLT buffer containing $\beta$-mercaptoethanol and vigorous shaking in a tissue blender. Lysates were stored at $-20^{\circ} \mathrm{C}$ (protein) or at $-80^{\circ} \mathrm{C}$ (RNA) until further processing.

Dot blotting and western blotting. Dot blots were prepared by spotting equimolar amounts of lfTSP (100 ng) and sfTSLP (50 ng) peptides on prewetted nitrocellulose membranes (Bio-Rad, Hercules, 
CA), after which the membranes were soaked in $4 \%$ bovine serum albumin for $1 \mathrm{~h}$ at room temperature. Antibodies were applied for $2 \mathrm{~h}$ at room temperature. The secondary antibody used was goat antirabbit IgG conjugated to alkaline phosphatase (Jackson ImmunoResearch Laboratories, West Grove, PA). Spots were visualized using the 5-bromo-4-chloro-3-indolyl phosphate/nitro blue tetrazolium substrate (Sigma-Aldrich). Protein lysates were mixed 1:3 with sample buffer containing dithiothreitol and $\beta$-mercaptoethanol (for lfTSLP detection) or 1:1 Tricine Sample Buffer (Bio-Rad) with $2 \% \beta$-mercaptoethanol (for sfTSLP detection) and boiled for $5 \mathrm{~min}$. Whole saliva was mixed 1:1 with sample buffer, and boiled for $10 \mathrm{~min}$. Concentration of protein in the lysates was determined using a protein assay (Bio-Rad) with $\gamma$-globulin as a standard protein. Thirty-five micrograms of protein lysate or $20 \mu \mathrm{l}$ of saliva sample was loaded onto 4-20\% (for lfTSLP detection) or $10-20 \%$ precast polyacrylamide gradient gels (Bio-Rad) (for sfTSLP detection) and subjected to sodium dodecyl sulfate-gel electrophoresis. Proteins were transferred to nitrocellulose membranes (Bio-Rad) (50 min; for lfTSLP detection) or polyvinylidene difluoride membranes (Bio-Rad) (15 min; for sfTSLP detection) and incubated in Protein Free Blocking buffer (Pierce Biotechnology) or $4 \%$ bovine serum albumin for $1 \mathrm{~h}$ at room temperature. LfTSLP and sfTSLP protein were detected using rabbit polyclonal anti-TSLP (no. 4021 (ProSci, Poway, CA; $1 \mu \mathrm{g} \mathrm{ml}^{-1}$ ) or ab47943 (Abcam; $\left.0.5 \mu \mathrm{g} \mathrm{ml}^{-1}\right)$ ), as indicated in the figures. The secondary antibody used was goat anti-rabbit IgG conjugated to alkaline phosphatase (Jackson IRL). Reactive bands were visualized using 5-bromo-4-chloro-3-indolyl phosphate/nitro blue tetrazolium substrate (Sigma-Aldrich). Densitometry was applied to some of the bands obtained after western blotting, by scanning the blotting membranes and using the gel analysis function in ImageJ (National Institute of Health, Bethesda, MD).

Immunohistochemistry. Biopsies were fixed in $4 \%$ buffered formaldehyde for $24 \mathrm{~h}$, dehydrated, and embedded in paraffin. Sections $(4 \mu \mathrm{m})$ were dewaxed, rehydrated, and quenched for endogenous peroxidase with $0.3 \% \mathrm{H}_{2} \mathrm{O}_{2}$ in methanol. Heat-induced epitope retrieval was performed in a $0.05 \%$ citraconic anhydride solution $(\mathrm{pH}$ 7.4 ) in distilled water in a pressure cooker (Biocare Medical, Concord, CA) at $100{ }^{\circ} \mathrm{C}$ for $15 \mathrm{~min}$. Sections were cooled, equilibrated in PBS, and blocked with $5 \%$ normal rabbit serum before overnight incubation at $4{ }^{\circ} \mathrm{C}$ with rabbit anti-human TSLP antibody, as indicated in the figures. As visualization systems, we used biotinylated goat anti-rabbit IgG antibody (Vector Laboratories, Burlingame, CA), followed by horseradish peroxidase-conjugated avidin-biotin complex (Vector Laboratories). Immunohistochemical staining was visualized using 3,3-diaminobenzidine (Sigma-Aldrich) as substrate, and hematoxylin was applied for counterstaining. Pictures were obtained using a Nikon Eclipse 90i microscope (Nikon Europe, Amsterdam, The Netherlands) equipped with a Plan Fluor $20 \times$ NA 0.50 objective (Figure 1d and Supplementary Figure S2), a Plan Fluor $10 \times$ NA 0.30 objective (Figure 1g), and a Nikon DS-Ri1 digital camera on the DS-L2 interface (Nikon, Tokyo, Japan). Images were captured with the NIS-Elements F software (Nikon; version 3.22.00) at a resolution of 3,840 $\times 3,072$ pixels per manual exposure with fixed shutter time, gain amplification, and illumination and at room temperature. Pictures were taken in one session, ensuring identical microscope, and camera settings. The images were opened in Adobe Photoshop (CS5, version 12.0.4) and mounted as displayed in the figures. Then, a separate layer was created that was used to adjust brightness and color balance on all photographs in the figure at the same time, to ensure that all adjustments for a given figure were made on all pictures collectively.

esiRNA knockdown. Cultured oral keratinocytes were transfected with esiRNA against TSLP (EHU075571) or scramble esiRNA (both from Sigma-Aldrich) with INTERFERin Transfection Reagent (Polyplus, Illkirch, France) according to the manufacturer's instructions. Transfection reactions were performed in extract-free keratinocyte serum-free medium (Gibco-BRL). Cell media were changed to extract-containing media $20 \mathrm{~h}$ after transfection. Cells were harvested $24 \mathrm{~h}$ after transfection for mRNA analysis and $48 \mathrm{~h}$ after transfection for protein analysis.

Real-time PCR. Total RNA was extracted from cell or biopsy lysates by the use of columns (RNeasy; Qiagen), according to the manufacturer's instructions. Quantity and purity of the RNA was measured using an ND-2000 spectrophotometer (NanoDrop Technologies, Wilmington, DE). Four hundred nanograms of RNA was reverse transcribed into cDNA using a Reverse Transcription Core Kit (RT-RTCK-05; Eurogentec, Seraing, Belgium) with random nonamers as RT primers and a Moloney murine leukemia virus reverse transcriptase enzyme (Eurogentec). Duplicate samples in Fast EvaGreen qPCR Master Mix (Biotium, Hayward, CA) were run on the MX4000 instrument (Stratagene, La Jolla, CA). The levels of TSLP mRNA were normalized with regard to the levels of the glyceraldehyde 3-phosphate dehydrogenase mRNA ( $\Delta \mathrm{Ct}$ method) and were calculated and displayed as $2^{-\triangle \mathrm{Ct}}$ values. For the esiRNA knockdown experiment, the level of sfTSLP mRNA was normalized with regard to E-cadherin. Primer sequences are given in Supplementary Table S1 (Sigma-Aldrich). DNA amplification was performed using the thermal cycling profile of initial denaturation at $95^{\circ} \mathrm{C}$ for $10 \mathrm{~min}$ followed by 40 cycles of amplification (denaturation at $95^{\circ} \mathrm{C}$ for $15 \mathrm{~s}$ and annealing and elongation for $1 \mathrm{~min}$ at $60^{\circ} \mathrm{C}$ ). Fluorescence data were collected during the annealing stage of amplification.

In situhybridization. Chromogen in situ hybridization was carried out on formaldehyde-fixed, paraffin-embedded human tissues using RiboMap and BlueMap Kits (Ventana Medical Systems, Illkirch, France) in the Ventana Discovery environment. A double digoxinlabeled LNA mRNA detection probe for sfTSLP was used $\left(5^{\prime}\right.$-TCA TAGGCGGCAAAGTTTACGA-3'; Exiqon, Vedbaek, Denmark). The probe was hybridized at $52{ }^{\circ} \mathrm{C}$ and amplified by using antidigoxin antibodies (Jackson IRL). Scramble probe was used as a control (Exiqon).

Antibacterial assay. Overnight bacterial cultures were spun down at $5,000 \mathrm{~g}$ for $10 \mathrm{~min}$, resuspended in $10 \mathrm{~mm}$ sodium phosphate buffer, and diluted to prepare $1 \mathrm{ml}$ of bacterial suspension $\mathrm{OD}_{600 \mathrm{~nm}}=0.350$, corresponding to $10^{6}$ per $\mathrm{ml}$ of $S$. mitis cells. This estimate was also used for the other bacteria. The $\mathrm{OD}_{600 \mathrm{~nm}} 0.350$ suspension was diluted 1:20 to get working suspensions $\left(5 \times 10^{5}\right.$ per $\left.\mathrm{ml}\right)$. Two hundred microliters of each bacterial working suspension was applied to wells in a 96-well plate giving $1 \times 10^{5}$ cells per well. Bacterial cultures were incubated with concentrations of sfTSLP, lfTSLP, and LL-37 as indicated in Figure 2a, b or without test substance, for $2 \mathrm{~h}$ at $37^{\circ} \mathrm{C}$ in air supplemented with $5 \% \mathrm{CO}_{2}$. At the end of the incubation, serial dilutions of each bacterial suspension $(25 \mu \mathrm{l})$ were plated on agar plates. After overnight incubation at $37^{\circ} \mathrm{C}$, the number of colonyforming units per $\mathrm{ml}$ was determined.

Analysis of STAT5 phosphorylation. STAT5 phosphorylation in response to sfTSLP and lfTSLP was examined in blood-derived CD1c ${ }^{+}$myeloid DCs. CD1c ${ }^{+}$DCs were isolated from fresh buffy coats using positive selection with magnetic beads (Miltenyi Biotec, Bergisch Gladbach, Germany) and incubated with poly(I:C) for $24 \mathrm{~h}$ at $37^{\circ} \mathrm{C}$ in $5 \% \mathrm{CO}_{2}$, to induce high levels of TSLP receptor expression. ${ }^{28}$ The next day, DCs were treated with IfTSLP or sfTSLP for $15 \mathrm{~min}$, or preincubated for $15 \mathrm{~min}$ with sfTSLP before incubation with lfTSLP for $15 \mathrm{~min}$, and subsequently fixed in $1.5 \%$ paraformaldehyde. The DCs were permeabilized in methanol before incubation with monoclonal rabbit antibodies to pSTAT5 (Tyr649) (Cell Signaling Technology, Danvers, MA), followed by Alexa 488-conjugated goat anti-rabbit secondary antibodies (Life Technologies, Grand Island, NY). Cells were analyzed on a BD LSRFortessa (BD Biosciences, Franklin Lakes, $\mathrm{NJ})$.

Statistical analysis. The paired $t$-test and repeated-measures analysis of variance on ranks (Friedman) with subsequent pairwise multiple 
comparison (Student-Newman-Keuls) were used to test differences between groups, using the Sigmaplot v 12.0 software (Systat Software, San Jose, CA). $P$-values of $<0.05$ were considered statistically significant.

SUPPLEMENTARY MATERIAL is linked to the online version of the paper at http://www.nature.com/mi

\section{ACKNOWLEDGMENTS}

We thank oral surgery practitioners Dr Karatsaidis (Galleri Oslo Klinikken), Dr Hals (Tannlegene i Bogstadveien 51, Oslo, Norway), and Dr Li (ENT Division, Oslo University Hospital) for providing biopsy material. We also thank H. Weidemann and C. Khuu, Department of Oral Biology, Dental Faculty, University of Oslo, Norway, for excellent technical assistance. The study was funded by the University of Oslo, Oslo, Norway.

\section{DISCLOSURE}

The authors declare no conflict of interest.

c) 2015 Society for Mucosal Immunology

\section{REFERENCES}

1. Friend, S.L., Hosier, S., Nelson, A., Foxworthe, D., Williams, D.E. \& Farr, A. A thymic stromal cell line supports in vitro development of surface $\operatorname{lgM}+B$ cells and produces a novel growth factor affecting $B$ and $T$ lineage cells. Exp. Hematol. 22, 321-328 (1994).

2. Soumelis, V. et al. Human epithelial cells trigger dendritic cell mediated allergic inflammation by producing TSLP. Nat. Immunol. 3, 673-680 (2002).

3. Kashyap, M., Rochman, Y., Spolski, R., Samsel, L. \& Leonard, W.J Thymic stromal lymphopoietin is produced by dendritic cells. J. Immunol. 187, 1207-1211 (2011).

4. Allakhverdi, Z. et al. Thymic stromal lymphopoietin is released by human epithelial cells in response to microbes, trauma, or inflammation and potently activates mast cells. J. Exp. Med. 204, 253-258 (2007).

5. Rochman, I., Watanabe, N., Arima, K., Liu, Y.J. \& Leonard, W.J Cutting edge: direct action of thymic stromal lymphopoietin on activated human CD4 + T cells. J. Immunol. 178, 6720-6724 (2007).

6. Ziegler, S.F., Roan, F., Bell, B.D., Stoklasek, T.A., Kitajima, M. \& Han, H. The biology of thymic stromal lymphopoietin (TSLP). Adv. Pharmacol. 66 , 129-155 (2013).

7. He, B. et al. Intestinal bacteria trigger T cell-independent immunoglobulin A(2) class switching byinducing epithelial-cell secretion of the cytokine APRIL. Immunity 26, 812-826 (2007).

8. Watanabe, N. et al. Hassall's corpuscles instruct dendritic cells to induce $\mathrm{CD} 4+\mathrm{CD} 25+$ regulatory $\mathrm{T}$ cells in human thymus. Nature 436 1181-1185 (2005).

9. lliev, I.D. et al. Human intestinal epithelial cells promote the differentiation of tolerogenic dendritic cells. Gut 58, 1481-1489 (2009).
10. Hanabuchi, S. et al. Thymic stromal lymphopoietin-activated plasmacytoid dendritic cells induce the generation of FOXP3 + regulatory T cells in human thymus. J. Immunol. 184, 2999-3007 (2010).

11. Sonesson, A. et al. Thymic stromal lymphopoietin exerts antimicrobial activities. Exp. Dermatol. 20, 1004-1010 (2011).

12. Harada, M. et al. Functional analysis of the thymic stromal lymphopoietin variants in human bronchial epithelial cells. Am. J. Respir. Cell. Mol. Biol. 40, 368-374 (2009).

13. Reche, P.A. et al. Human thymic stromal lymphopoietin preferentially stimulates myeloid cells. J. Immunol. 167, 336-343 (2001).

14. Rimoldi, M. et al. Intestinal immune homeostasis is regulated by the crosstalk between epithelial cells and dendritic cells. Nat. Immunol. 6, 507-514 (2005).

15. Quentmeier, H. et al. Cloning of human thymic stromal lymphopoietin (TSLP) and signaling mechanisms leading to proliferation. Leukemia 15, 1286-1292 (2001).

16. Smelter, D.F., Sathish, V., Thompson, M.A., Pabelick, C.M., Vassallo, R. \& Prakash, Y.S Thymic stromal lymphopoietin in cigarette smoke-exposed human airway smooth muscle. J. Immunol. 185, 3035-3040 (2010).

17. Harder, J., Schröder, J.M. \& Gläser, R The skin surface as antimicrobial barrier: present concepts and future outlooks. Exp. Dermatol. 22, 1-5 (2013).

18. De Smet, K. \& Contreras, R Human antimicrobial peptides: defensins, cathelicidins and histatins. Biotechnol. Lett. 27, 1337-1347 (2005).

19. Aas, J.A., Paster, B.J., Stokes, L.N., Olsen, I. \& Dewhirst, F.E Defining the normal bacterial flora of the oral cavity. J. Clin. Microbiol. 43, 5721-5732 (2005).

20. Lai, Y. et al. Activation of TLR2 by a small molecule produced by Staphylococcus epidermidis increases antimicrobial defense against bacterial skin infections. J. Invest. Dermatol. 130, 2211-2221 (2010).

21. Kropec, A., Hufnagel, M., Zimmermann, K. \& Huebner, J In vitro assessment of the host response against Enterococcus faecalis used in probiotic preparations. Infection 33, 377-379 (2005).

22. Alteri, C.J. \& Mobley, H.L Escherichia coli physiology and metabolism dictates adaptation to diverse host microenvironments. Curr. Opin. Microbiol. 15, 3-9 (2012).

23. Tran, S.L. et al. Haemolysin II is a Bacillus cereus virulence factor that induces apoptosis of macrophages. Cell. Microbiol. 13, 92-108 (2011).

24. Dale, B.A. \& Fredericks, L.PAntimicrobial peptides in the oral environment: expression and function in health and disease. Curr. Issues Mol. Biol. 7 , 119-133 (2005).

25. Nomura, K. et al. Regulation of interleukin-33 and thymic stromal lymphopoietin in human nasal fibroblasts by proinflammatory cytokines. Laryngoscope 122, 1185-1192 (2012).

26. Hung, T.J. et al. shRNA for thymic stromal lymphopoietin: a novel therapeutic approach for pulmonary fibrosis. J. Cell. Sci. Ther. 4, 144 (2013).

27. Storesund, T., Hayashi, K., Kolltveit, K.M., Bryne, M. \& Schenck, K. Salivary trefoil factor 3 enhances migration of oral keratinocytes. Eur. J. Oral Sci. 116, 135-140 (2008).

28. Lu, N., Wang, Y.H., Wang, Y.H., Arima, K., Hanabuchi, S. \& Liu, Y.J. TSLP and IL-7 use two different mechanisms to regulate human CD4 + T cell homeostasis. J. Exp. Med. 206, 2111-2119 (2009). 